\title{
FAILED ANTERIOR RECONSTRUCTION FOR SHOULDER INSTABILITY
}

\author{
ROBERT H. HAWKINS, RICHARD J. HAWKINS
}

\author{
From Shaughnessy Hospital, Vancouver and St Joseph's Hospital, London, Canada
}

\begin{abstract}
We report a retrospective study of $\mathbf{4 6}$ patients with continuing difficulties after anterior reconstruction of a shoulder for instability. In 31 patients instability was still present; in 12 of these, posterior or multidirectional instability had not been recognised and a further 11 had an uncorrected anatomical defect. In 20 patients with significant pain there was often more than one cause: impingement syndrome was seen in nine, osteoarthritis in seven, implant irritation in four and instability alone in two. A disabling medial rotation contracture was seen in 10 patients, four of whom had painful osteoarthritis. We conclude that recurrence of symptoms may imply that the direction of the instability was not recognised, that an anterior repair should not be too tight, and that pain after successful stabilisation is often due to impingement.
\end{abstract}

Glenohumeral instability has long been recognised as a major cause of disability, especially among athletes. Recurrent dislocations in anterior, posterior or inferior directions are described in earlier writings (DePalma 1983). More recently, most attention has focused on recurrent anterior dislocation, for which many different surgical procedures have been reported; those described by Bankart, Magnuson-Stack, Putti-Platt, Bristow and $\mathrm{Du}$ Toit remain popular (Moseley 1945). When instability persists after one of these procedures, it is frequently presumed to be due to recurrence of anterior dislocation. However, recent studies of glenohumeral instability (Neer and Foster 1980; Gerber and Ganz 1984) suggest that subsequent operations may often have been based upon an incomplete or an incorrect diagnosis; other causes of failure are now becoming evident. We report a retrospective study of 46 cases of failed anterior reconstructions of the shoulder and attempt to analyse the reasons for persistence of the instability.

\section{MATERIAL AND METHODS}

Successful operation for anterior instability should restore stability while maintaining a painless functional range of movement. Failure may be caused by recurrence of instability, significant postoperative pain or marked loss of movement. Forty-six patients with one or more of these problems were referred to us by other surgeons for

R. H. Hawkins, MD, FRCS (C), Clinical Instructor in Orthopaedic Surgery

University of British Columbia, Vancouver, Canada.

R. J. Hawkins, MD, FRCS (C), FACS, Associate Professor of Orthopaedic Surgery

University of Western Ontario, London, Canada.

Requests for reprints should be sent to Dr Robert H. Hawkins, Shaughnessy Hospital Pavilion, G627, 4500 Oak Street, Vancouver, British Columbia V6H 3N1, Canada.

(c) 1985 British Editorial Society of Bone and Joint Surgery

$0301-620 \times / 85 / 5154 \$ 2.00$

VOL. 67-B, No. 5, NOVEMBER 1985 assessment and treatment; they form the basis of this report.

All 46 patients had problems after their initial anterior reconstruction; all were evaluated by us and subsequently treated under our direction. Revision operations were performed on 27 patients. Clinical details of all patients are summarised in Table $\mathrm{I}$.

\section{CAUSES OF FAILURE}

The cause of failure was persisting instability in 31 cases, significant pain in 20, and marked loss of mobility in 10 . Many cases had more than one cause of failure.

Persisting or renewed instability. Three separate categories were distinguished among the 31 in this group. The first consisted of eight patients with recurrence of anterior instability after a major re-injury; for these patients, the original diagnosis was probably correct and the original procedure appropriate, but the violence of the new injury broke down what had been a satisfactory repair.

The second group comprised 11 patients with recurrence of anterior instability after a minor injury. Here the original diagnosis may have been correct but the operative procedure was inadequate.

The third group were those whose instability was either posterior (two patients) or multidirectional (10 patients). For these 12 we felt that the original diagnosis had probably been incomplete or incorrect and the original procedure therefore inappropriate.

Postoperative pain. This was a major problem for 20 patients, and we found five underlying causes (Table II). Nine patients had subacromial impingement syndrome, and seven of them later had a successful anterior acromioplasty (Neer 1972). Seven patients had painful osteoarthritis, and in six of these it seemed to be associated with a tight anterior repair; the seventh (Case 25) had intra-articular staples and sustained severe articular damage from recurrence during a seizure. Irritation by 
$\|1\|\|\|\|\| \|$

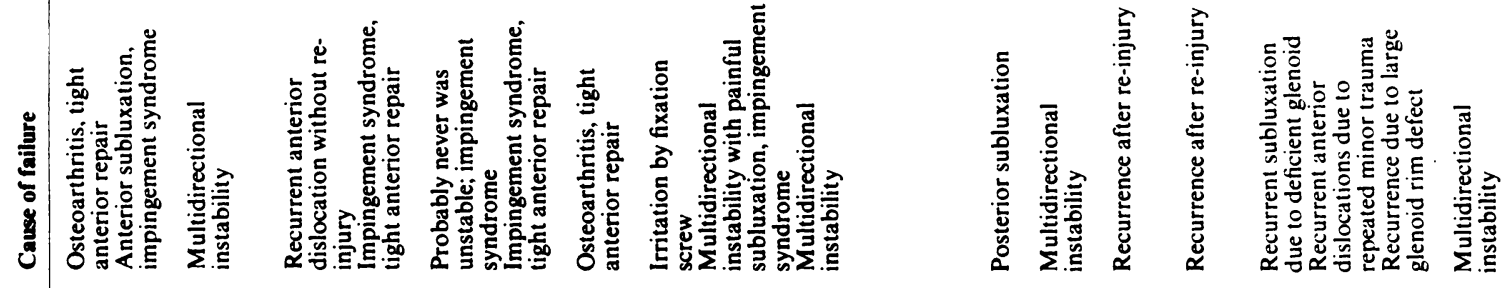

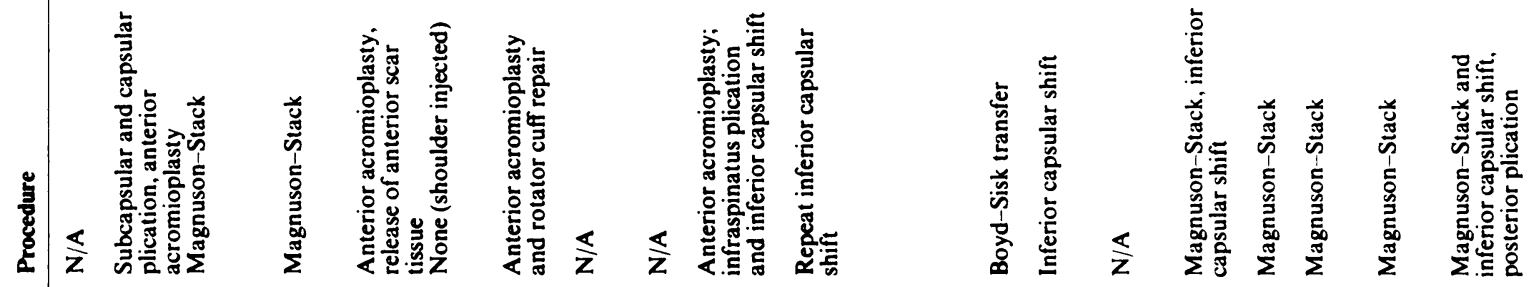
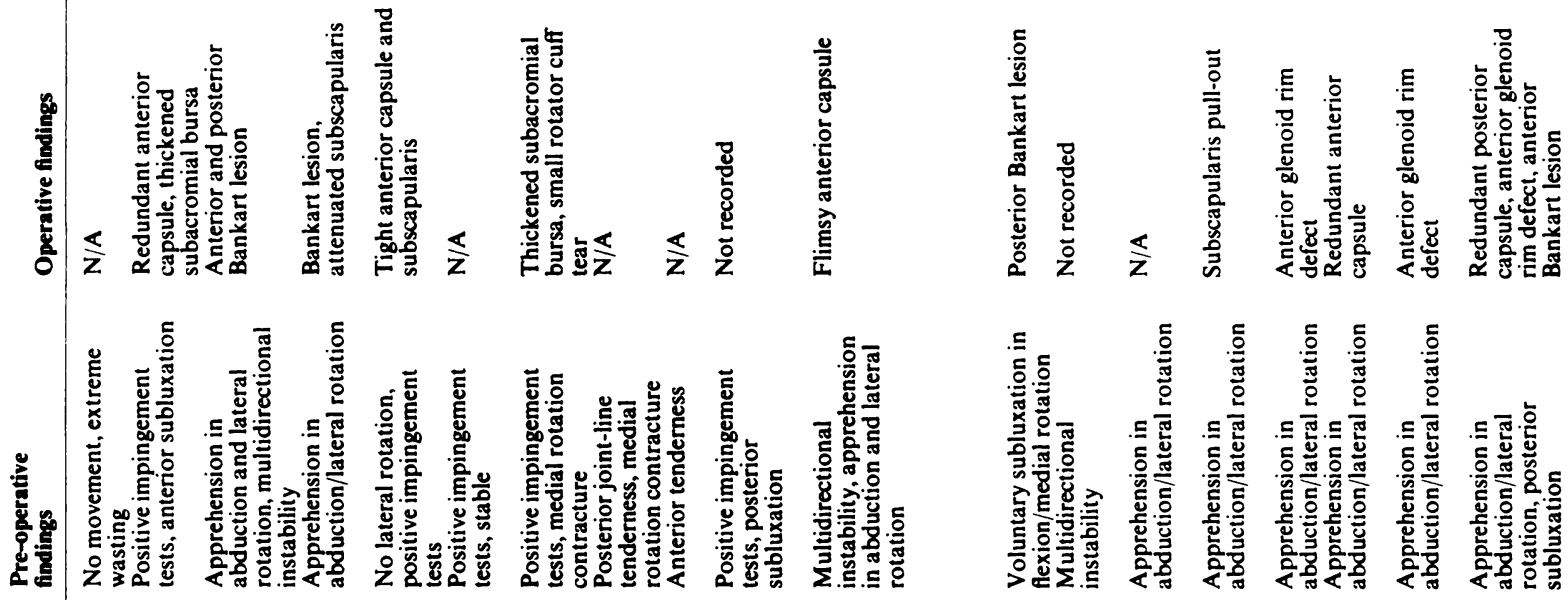

离

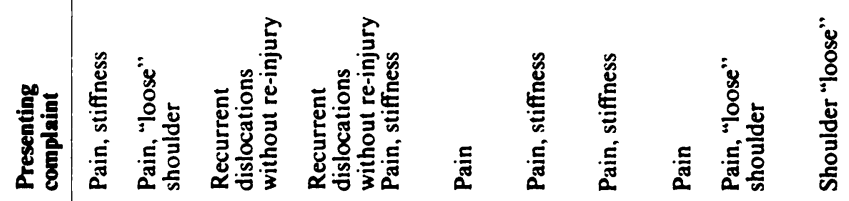

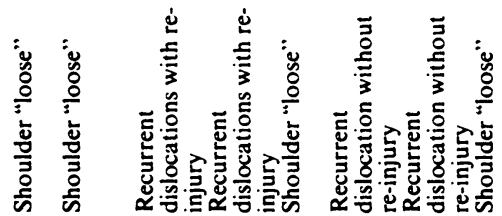

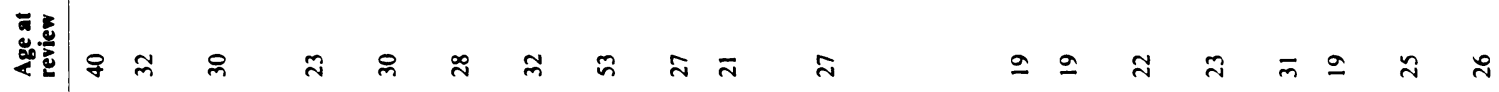

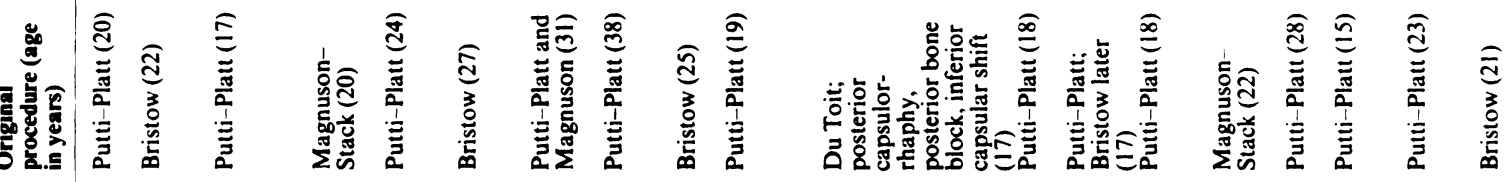

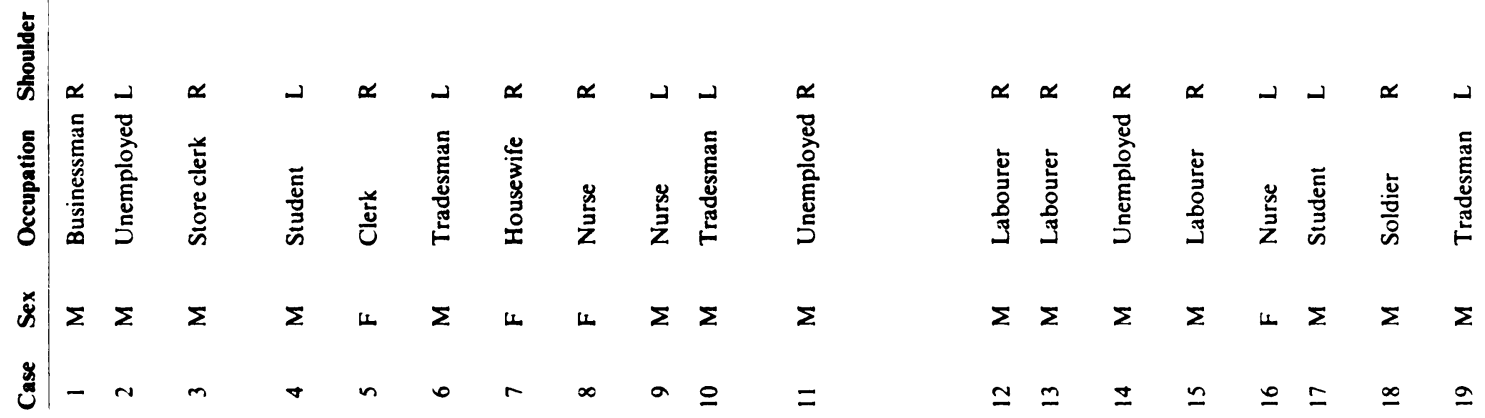




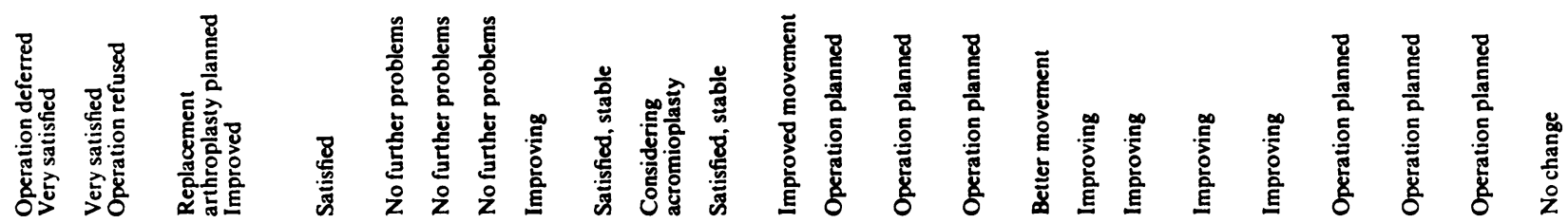

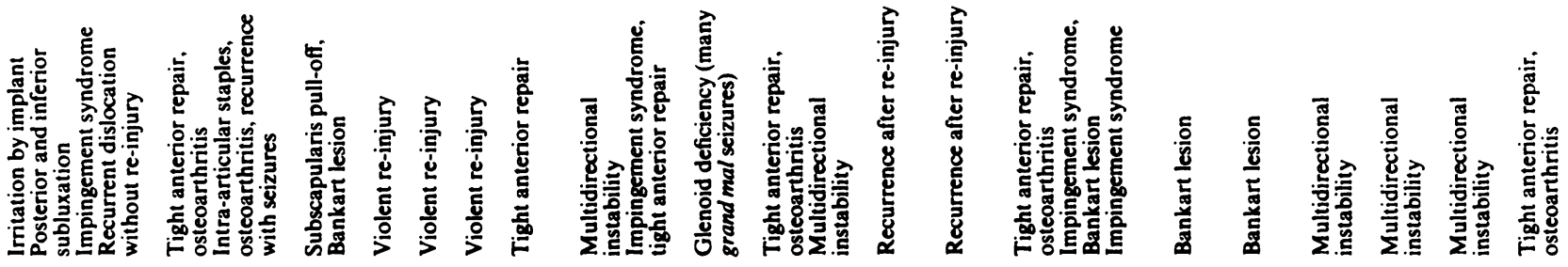

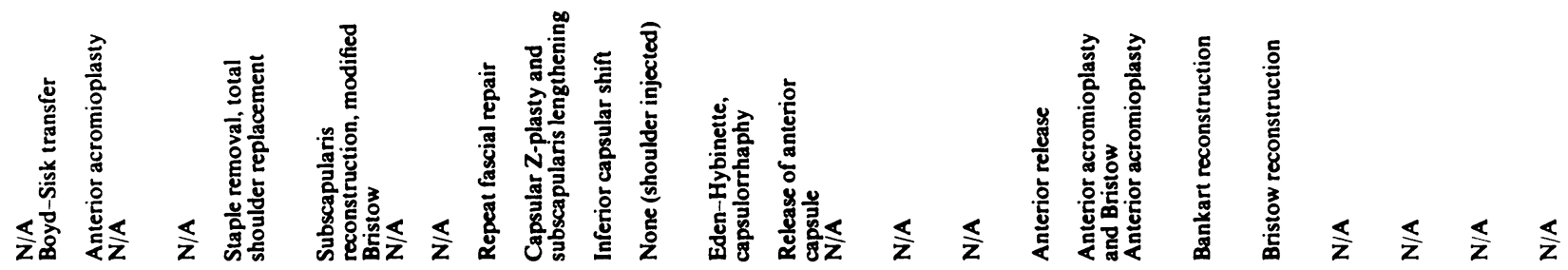

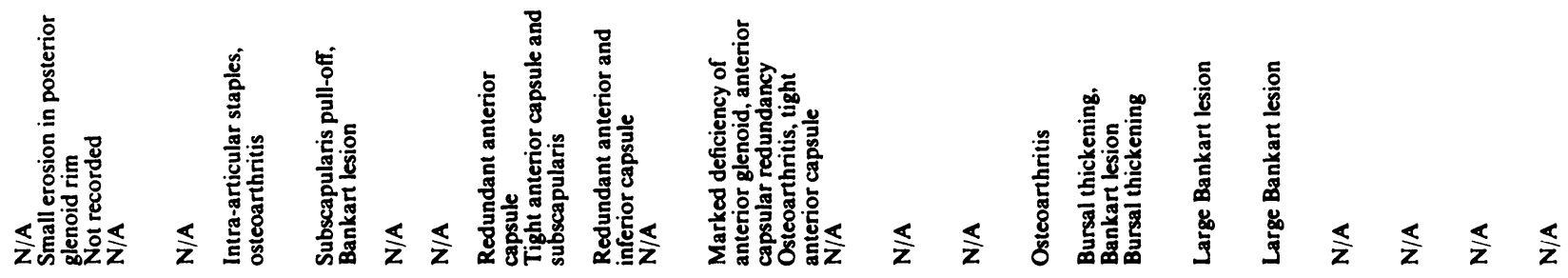

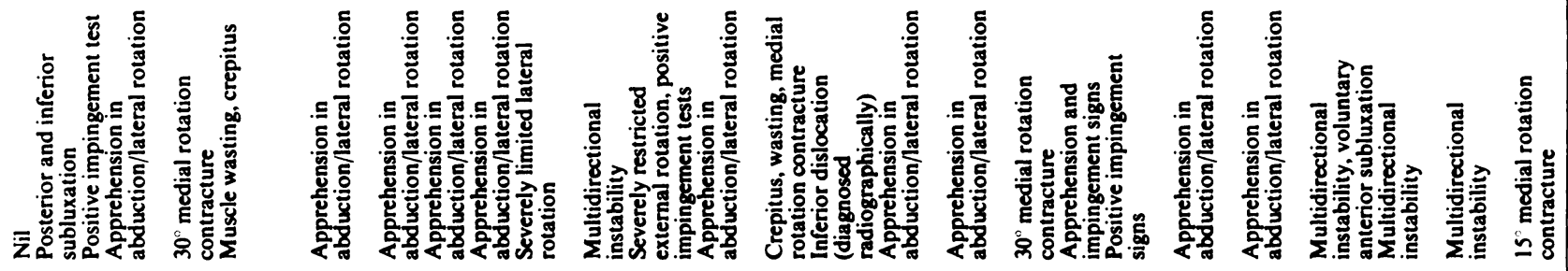

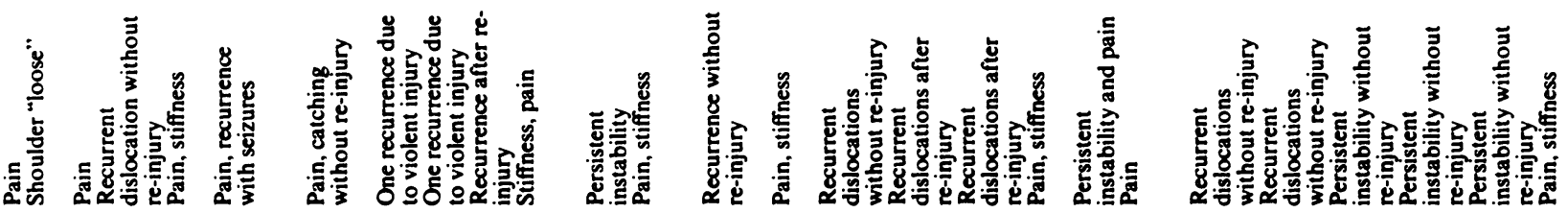

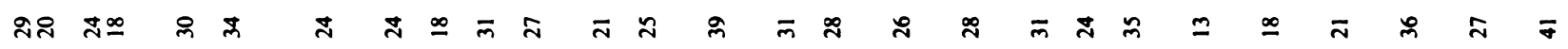

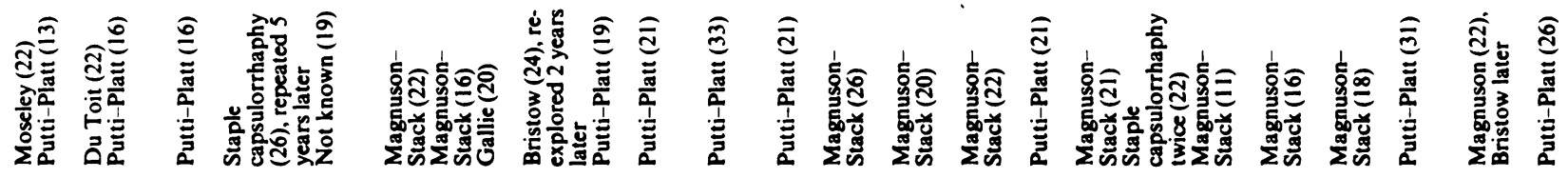

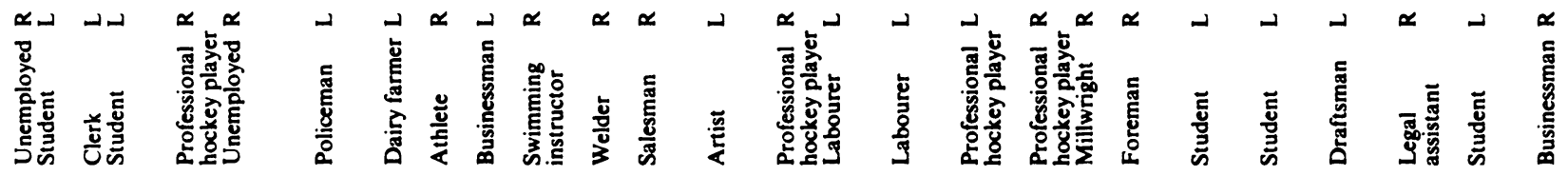

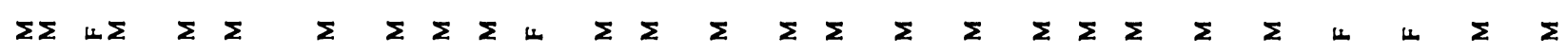

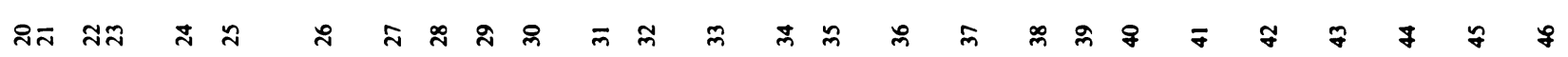


Table II. Five causes for persisting postoperative pain in 20 patients

\begin{tabular}{ll}
\hline Impingement syndrome & 9 \\
Osteoarthritis & 7 \\
Irritation by metallic implant & 4 \\
Painful stiffness & 1 \\
Painful instability & 2 \\
\hline
\end{tabular}

an implant was diagnosed in four patients, all of whom had intra-articular screws or staples. Pain from either stiffness alone or from painful instability accounted for only three cases.

Restricted mobility. This was a predominant cause of failure in 10 patients. We listed this category separately, although all cases of limited mobility also had pain. All were associated with a tight anterior repair. One patient had pain at the site of the repair (Case 30), three had an accompanying impingement syndrome, and six had significant osteoarthritis.

\section{DISCUSSION}

In treating shoulder instability, the two aims of reconstructive surgery are to restore stability and to retain mobility without pain. In the past, surgeons have concentrated on stability; they have selected the simplest procedure which achieves that objective and which also has a low recurrence rate. Several procedures satisfy both criteria, and therefore remain popular: these include the Magnuson-Stack (Rothman, Marvel and Heppenstall 1975), the Putti-Platt (Leach et al. 1982), the Du Toit (Sisk and Boyd 1974), and the Bristow operations (Hill et al. 1981).

Glenohumeral instability exists in many forms and may be classified according to its degree (subluxation or dislocation); the circumstances in which it occurs (acute, chronic, recurrent, voluntary and habitual); the initial cause (traumatic or atraumatic); and the direction in which the humeral head displaces (anterior, posterior, inferior and multidirectional) (Matsen and Zuckerman 1983). Recurrent anterior subluxation with little trauma is now considered to be more common than was once thought (Hastings and Coughlin 1981; Warren 1983); posterior instability (Hawkins, Koppert and Johnston 1984) and multidirectional instability (Neer and Foster 1980) have also received recent attention.

The stability of the shoulder is maintained by three different factors which work together to provide an extraordinary range of movement and yet to maintain a stable ball and socket joint. Bone and capsuloligamentous structures provide static stability while dynamic stability is conferred by the muscles of the rotator cuff. In the normal shoulder, no single structure is responsible for stability in all ranges of glenohumeral movement (Turkel et al. 1981). It seems that no single "essential lesion" is responsible for recurrent anterior instability.

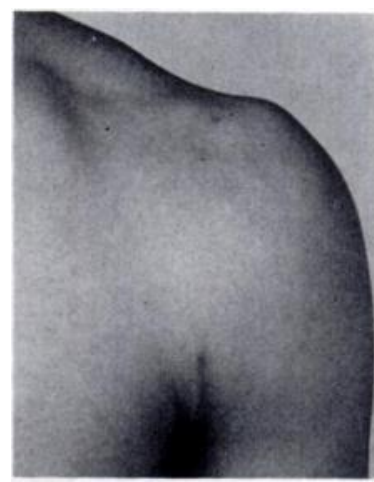

Fig. 1

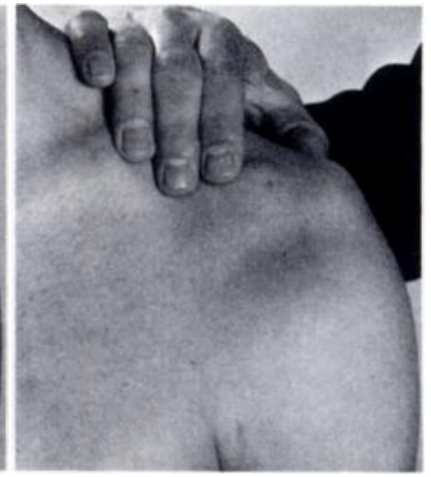

Fig. 2
Case 10. This patient presented with a painful and "loose" shoulder three years after a Putti-Platt operation. Downward traction shows the sulcus sign of inferior subluxation.

Significant causes may include glenoid defects of more than one-third of the joint surface; capsular avulsions greater than a centimetre in length; and capsular deficiency or subscapularis laxity, especially when there is no bone defect or capsular detachment (Matsen and Zuckerman 1983). The concept of "anterior capsular mechanism", first suggested by Townley (1950), may still have some place in explaining instability.

Current methods for dealing with instability include both conservative and operative treatment. Non-operative treatment includes giving advice on avoiding certain movements and prescribing exercises to strengthen the medial and lateral rotator muscles; an external checkrein of adhesive tape or an abduction harness may also be considered. Operations for recurrent anterior instability may aim to provide some form of internal check-rein which prevents the joint reaching a position of risk, to achieve the same object by a rotation osteotomy, or to identify and correct any anatomical faults.

We analysed persistent instability in 31 patients. In eight, recurrence followed an injury severe enough to break down a satisfactory repair, while in 11 uncorrected pathology was thought to be responsible. Lesions seen at re-operation included capsular detachments from the anterior glenoid rim, redundant anterior capsule, and deficiency of the bony glenoid. It is clearly important that any anatomical deficiencies should be identified and corrected at the first operation. In a further 12 patients there had been failure to recognise that the patient had multidirectional or posterior instability. The need for a correct initial diagnosis is obvious.

The second major group of failures were those with significant pain after stabilising procedures (Figs 1 and 2). Impingement syndrome, the commonest cause in this group, may occur independently of instability or in association with it; the syndrome can, of course, be readily diagnosed and treated (Hawkins and Kennedy 1980).

The development of painful osteoarthritis after a stabilising procedure is especially disturbing. Of the seven cases in our series, one was related to a staple in the joint. In the other six, osteoarthritis was seen an average 


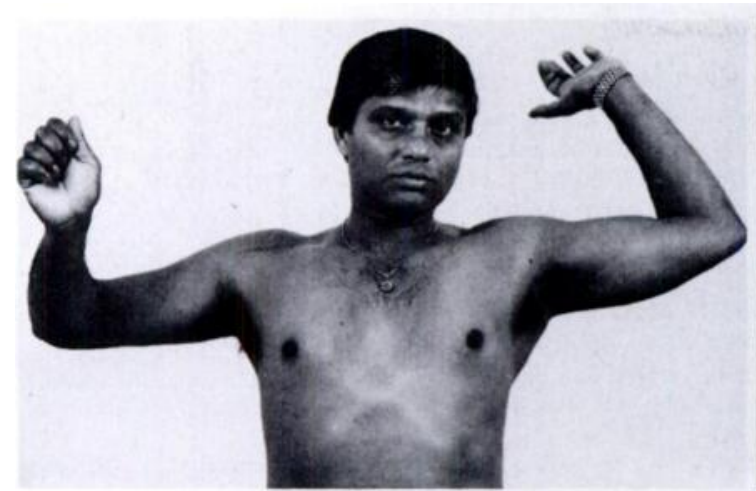

Fig. 3

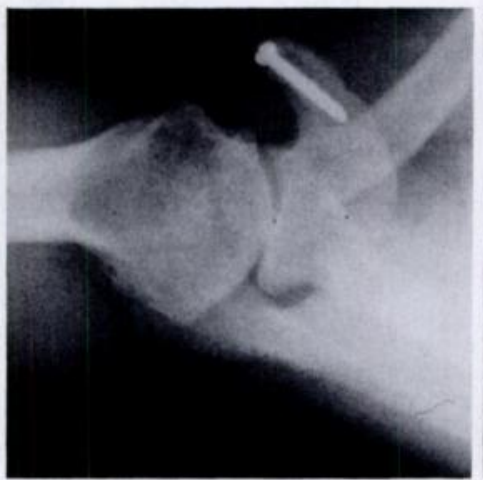

Fig. 4

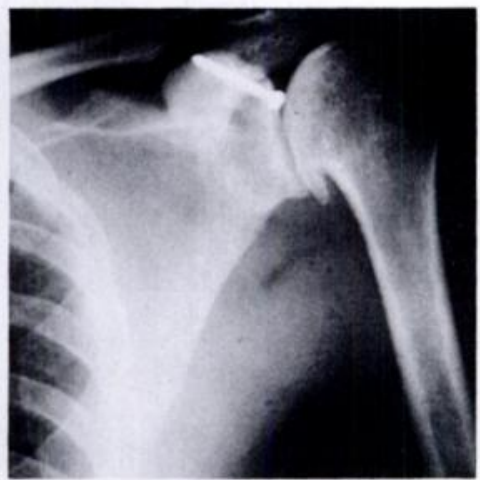

Fig. 5

Case 46. Reviewed 15 years after a Putti-Platt repair which was too tight, this man has a marked medial rotation contracture of the right shoulder. Figures 4 and 5 show advanced degenerative arthritis.

of 13 years after the primary operation, and the only associated factor was a tight anterior repair (Figs 3 to 5). These patients could all remember vain attempts to regain movement, particularly lateral rotation. We believe that an excessively tight repair may provoke significant glenohumeral arthritis, especially in a joint which has already been damaged by recurrent instability.

The third and last major group of failures included those with significant restriction of movement. It is accepted that any repair may restrict mobility to some extent, but in the present review significant loss of movement was the principal complaint of 10 patients; the Putti-Platt reconstruction accounted for nine of these, and six of the nine had osteoarthritis. These patients had paid a high price for gaining stability of their shoulder.

Our present approach to the management of glenohumeral instability is based on an appreciation of the causes of failure. We begin with a review of the circumstances in which displacement usually takes place so that we can advise the patient which activities should be avoided. All patients start a programme of exercises to strengthen the medial and lateral rotators, and the supraspinatus muscle. Occasionally a harness to limit abduction may be appropriate.

If these measures fail to control instability, then surgical repair is considered. A pre-operative assessment is made to identify the directions of instability and to determine any associated pathology such as generalised laxity, bony defects, rotator cuff tears and neuromuscular disorders. With this information, the surgeon can discuss alternatives with the patient.

If operation is undertaken, then under anaesthesia both shoulders are carefully tested for stability, with particular attention to direction(s) of displacement and to crepitus. The joint is exposed through a standard anterior approach and again examined fully, both to confirm the direction(s) of instability and to identify any anatomical deficiencies. The methods used for repair or reconstruction depend upon the pathology. Bankart lesions longer than one centimetre require reattachment. We treat capsuloligamentous laxity, especially if it is the only abnormality, by advancing the medial flap laterally to restrict lateral rotation, but take care to retain at least 25 of lateral rotation as tested with the elbow at the side. If significant antero-inferior instability is noted, an inferior capsular shift is performed (Neer and Foster 1980).

Postoperatively the arm is held in a sling for the first month, with the addition of a shoulder yoke to support the weight of the arm if an inferior capsular shift has been performed. In the second month limited movement is begun, together with isometric strengthening exercises. In the third month, the range of movement is extended and the exercises become isotonic. Contact sports are prohibited for the first six months.

Conclusions. On the basis of our analysis of failures, the following conclusions have been drawn:

1. An accurate diagnosis is essential and is based upon a careful history and physical examination followed by appropriate radiography; if operation is undertaken, then the first step is examination under anaesthesia.

2. The operative approach must be wide enough to allow full inspection of the pathology and any corrective procedures.

3. Screws or staples in or near the joint should be avoided.

4. Excessive limitation of lateral rotation should be avoided; this is a significant cause of a poor functional result and may be responsible in part for the later development of glenohumeral arthritis.

5. A common cause of postoperative pain is an impingement syndrome. 


\section{REFERENCES}

DePalma AF. Surgery of the shoulder. 3rd ed. Philadelphia: J. B. Lippincott, 1983:524.

Gerber C, Ganz R. Clinical assessment of instability of the shoulder: with special reference to anterior and posterior drawer tests. $J$ Bone Joint Surg [Br] 1984;66-B:551-6.

Hastings DE, Coughlin LP. Recurrent subluxation of the glenohumeral joint. Am J Sports Med 1981;9:352-5.

Hawkins RJ, Kennedy JC. Impingement syndrome in athletes. Am J Sports Med 1980;8:151-8.

Hawkins RJ, Koppert G, Johnston G. Recurrent posterior instability (subluxation) of the shoulder. J Bone Joint Surg $[\mathrm{Am}] 1984 ; 66-A$ : $169-74$.

Hill JA, Lombardo SJ, Kerlan RK, et al. The modification BristowHelfet procedure for recurrent anterior shoulder subluxations and dislocations. Am J Sports Med 1981;9:283-7.

Leach RE, Corbett M, Schepsis A, Stockel J. Results of a modified Putti-Platt operation for recurrent shoulder dislocations and subluxations. Clin Orthop 1982;164:20-5.

Matsen FA III, Zuckerman JD. Anterior glenohumeral instability. Clin Sports Med 1983;2(2):319-38.
Moseley HF. Shoulder lesions. Springfield, Illinois: Charles C. Thomas, 1945.

Neer CS II. Anterior acromioplasty for the chronic impingement syndrome in the shoulder. J Bone Joint Surg $[\mathrm{Am}] 1972 ; 54-A: 41-50$.

Neer CS II, Foster CR. Inferior capsular shift for involuntary inferior and multidirectional instability of the shoulder: a preliminary report. J Bone Joint Surg [Am] 1980;62-A:897-908.

Rothman RH, Marvel JP Jr, Heppenstall RB. Recurrent anterior dislocation of the shoulder. Orthop Clin North Am 1975;6(2):415-22.

Sisk TD, Boyd HB. Management of recurrent anterior dislocation of the shoulder: Du Toit-type or staple capsulorrhaphy. Clin Orthop 1974;103:150-6.

Townley CO. The capsular mechanism in recurrent dislocation of the shoulder. J Bone Joint Surg [Am] 1950;32-A:370-80.

Turkel SJ, Panio MW, Marshall JL, Girgis FG. Stabilizing mechanisms preventing anterior dislocation of the glenohumeral joint. $J$ Bone Joint Surg $[$ Am $] 1981 ; 63-A: 1208-17$.

Warren RF. Subluxation of the shoulder in athletes. Clin Sports Med $1983 ; 2(2): 339-54$. 